\title{
How to control a temporary DIDD based observatory in the field?
}

\author{
András Csontos ${ }^{1}{ }^{\star}$, Danijel Šugar ${ }^{2}$, Mario Brkić ${ }^{2}$, Péter Kovács ${ }^{1}$, László Hegymegi ${ }^{1}$ \\ ${ }^{1}$ Eötvös Loránd Geophysical Institute, Budapest, Hungary \\ ${ }^{2}$ University of Zagreb, Faculty of Geodesy, Institute for Geomatics, Zagreb, Croatia
}

\author{
Article history \\ Received October 14, 2011; accepted June 27, 2012. \\ Subject classification: \\ Instruments and techniques, Main geomagnetic field, Geomagnetic field variations and reversals, Magnetic anomalies, Magnetic storms.
}

\begin{abstract}
One of the main challenges on the course of the repeat station surveys is to determine the spatial differences of the geomagnetic elements between the repeat stations and the reference observatory. The difficulty arises from the fact, that the directly obtained differences are affected not only by spatial but also by temporal effects of external origin. The error deriving from the external effects can be efficiently diminished by the installation of an on-site vector variometer. In this case the spatial difference can be computed for night-time period, when the external field is less varying (both spatially and temporally) than during daytime. Installation of the on-site variometer in the field requires the fulfillment of nearly the same conditions as in the observatories, i.e. the control of the reference frame, the scale factors, the offsets, and the temperature effects of the magnetometer. The principle of the fluxgate and DIDD magnetometers is quite different from each other, therefore the two devices provide different possibilities to obtain accurate result. The paper discusses some of the possible instrumental errors and offers a method based on the DIDD technology for the determination of the reference frame of a portable recording station. We analyse real records measured during the joint Hungarian-Croatian repeat station survey.
\end{abstract}

\section{Introduction}

Geomagnetic observatories provide high quality records of the magnetic field elements continuously, with high temporal resolution. The density of the observatories however is not enough to monitor the relevant variation of the internal magnetic field in spatial scale. Therefore, decades ago, repeat station (RS) networks were established in the different countries with the aim of periodically monitoring the secular magnetic variation in uniform spatial distribution.

During the reoccupations of repeat stations a series of absolute magnetic data are produced. The measurements are carried out according to the recommendations of IAGA and MagNetE. In order to be able to temporally compare the secular data measured in different epochs, the measurements are reduced to common epoch using the permanent record of a reference observatory. On the course of the temporal reduction it is assumed that (1) the secular variations and (2) the transient magnetic variations are identical at the repeat station and the reference observatory [Newitt et al 1996 , Korte 2001]. The first assumption is generally valid if the time between the reoccupation of the secular station and the epoch of reduction is less than half a year. The identity of the transient variations of external origin however is seldom satisfied. The spatial and temporal dependence of the transient field variations are basically affected by the following regularities:

a) the variation of the north magnetic component increases with the geomagnetic latitude;

b) the spatial variation of the east transient magnetic field is basically affected by the longitudinal distance from the reference observatory;

c) recombination of the ring current developing during geomagnetic storms can result in long-lasting (typically more than 3 days) drift in the differences of corresponding components measured in different sites;

d) the variation of the vertical transient field is influenced by the spatial variation of the electrical conductivity of the subsurface through the telluric currents;

e) near the seaside extra magnetic field is produced by high conductivity contrast between of the ocean and the mainland;

f) the tectonomagnetic effect caused by mechanical stress may result in extra magnetic field in certain sites as compared to the reference observatory.

The influences of the most part of the above effects on the accuracy of the temporal correction can be minimized by completing the magnetic secular measurements in quiet geomagnetic conditions, i.e. in periods void of geomagnetic storms and in night-time when the solar quiet magnetic variation is the most stable. It must be emphasized however that some of the listed magnetic phenomena (e.g. tectonomagnetic variation) are independent from the geomagnetic circumstances, thus their effects in the temporal corrections are completely unavoidable.

Nowadays, the practice of the field absolute measurements are based on the absolute instruments (DIM, PPM), 
since they are basically free from calibration errors and temperature effects (at least theoretically). The automatic operation of the DIM device for providing permanent declination and inclination values in a longer period however is not yet applied in the field.

The condition for the quiet-time observation can thus be rarely satisfied in the practice of the repeat station campaigns, since the measurements must be carried out in daytime when the bearing azimuths are visible. To extend the observations to the more quiet night-time period it is suggested then to install a tri-component portable variometer in the vicinity of the repeat station. The closeness is necessary for ensuring the identity of the geomagnetic variation in the repeat station and the variometer site. By establishing the basis of the variometer through the absolute measurements the variometer record can be referenced to the repeat station.

Thus, the station can function as a temporary observatory, provided that the setup of the variometer is prudently completed and the accuracy parameters (e.g. orientation, scale factors, temperature of the device) of the instrument are continuously controlled.

In this paper we present the most important behaviours of two classical observatory variometers, i.e. the fluxgate and DIDD magnetometers. We argue that for field use the DIDD is superior than the fluxgate instrument. For this reason, we summarize the way of installation of the DIDD device in field environment and provide method for the determination of its calibration parameters. Finally, the calibration results are shown in case studies carried out in the Tihany observatory and in repeat stations of Hungary and Croatia.

\section{The fluxgate magnetometer}

The principle and the operation of the fluxgate (FGE) instrument are described by Jankowski and Sucksdorff [1996]. The biggest advances of the fluxgate devices are the low noise and the relatively high frequency response. For the calibration of the device twelve parameters have to be determined (three offsets, three scale factors, three orthogonality errors of the sensors and three orientation errors).

Marsal et al. [2009] described the on-site calibration procedure of the FGE magnetometer. From this it is evident that the completion of the whole process is unrealistic during the reoccupation of a repeat station. Namely, the absolute measurements made in the field survey can only calibrate the offset values of the FGE instrument, while the possible tilt of the sensor modifying the orientation of the device as well as the scale factor of the instrument remain undetermined. The usual comparison of the total field values measured by the PPM instrument and calculated from the three-component variometer record may also help to further correct the variometer data. However, since the contribution of the $\mathrm{Y}$ magnetic field in the calculated total field is practically negligible, the sensitivity of the above method is very limited regarding especially to the east component.

The fluxgate devices need a priori and a posteriori check in a reference observatory. During this process the data of the test instrument is compared with the observatory records during a certain period [Newitt et al. 1996]. It is noted that this procedure is necessary but still not enough for satisfying the requirements of an exact calibration.

The temperature effects of fluxgate magnetometers were presented by Newitt et al. [1996] in detail. Our temperature tests in the Tihany Geophysical Observatory prove that the most of the fluxgate magnetometers have large temperature coefficients and their behaviour depends significantly on the amplitude of the temperature change. Thus, the determination of a general temperature coefficient, as a correction factor is very limited [Csontos et al. 2007].

There are further possible sources of errors of the fluxgate devices, such as different type of orientation errors, orthogonality errors, voltage dependence of electronics, etc. Usually, these effects appear simultaneously. Unfortunately, there are no general solution for separating these errors and correct the dataset afterwards. For the case of the data reduction, the errors caused by the unknown calibration parameters of a fluxgate device may be occasionally higher than that caused by the non-identical external field variation between the station and reference observatory. Anyway, the recommendations of the book Guide for magnetic repeat station survey [Newitt et al. 1996] provide a useful help how to reach the best result with fluxgate magnetometers.

\section{The DIDD system and its operation}

3.1. The technical details of the DIDD system with its theoretical background

The DIDD instrument consists of a double coil system whose axes are perpendicular to each other. The axis of the $\mathrm{D}$ coil is horizontal and lies nearly at right angle to the local mean magnetic N-S direction, while the I coil axis lies in the local mean meridian plane nearly at right angle to the magnetic field. In the geometrical center of the system there is a sensor capable of measuring the total magnetic field. On the course of the operation of the DIDD parallel and antiparallel currents of same strength are introduced into each of the coils producing deflection field of about $10000 \mathrm{nT}$ along the axis of the two coils. From the values of the deflected total field readings, the declination and inclination components can be concluded. The prototype of this system, designed in the late 1960 's, used a Helmholtz coil system with a proton or optically pumped Rubidium and Cesium sensor.

Cooperation on a spherical coil, approximately $30 \mathrm{~cm}$ in diameter, began in the 1990s between ELGI and USGS. The product of this joint work was a molded spherical coil system made from epoxy resin. The development of the Overhauser magnetometers enabled to construct the fast DIDD system. 
An ideal solution is GEM's Overhauser magnetometer with a built-in current generator. The cycle time of one reading is 1 or 0.5 second and the resolution of the Overhauser magnetometer is $0.01 \mathrm{nT}$ [Csontos 2001].

A new Overhauser sensor with $150 \mathrm{~mm}$ in diameter was developed in 2002. For this sensor B. Heilig modeled a new spherical coil system which had a diameter of $200 \mathrm{~mm}$. This size was sufficiently small to construct a suspension device and to build a user friendly magnetometer. The demand for the field use of the system required the development of the MAGLIN data acquisition software enabling the remote control and remote checking of the data record via internet. The software was running under Linux operation system. This solution was flexible and stable enough to run on industrial PC which needed only $5 \mathrm{~W}$. The total consumption of the electronics of the DIDD, the GPS receiver and the PC was not more than $10 \mathrm{~W}$ so a $88 \mathrm{Ah}$ battery was far enough for the period of a short-term (maximum a week) reoccupation.

The phases of the development and tests of the instrument were repeatedly presented [see, e.g., Csontos et al. 2001, Hegymegi et al. 2004, Csontos et al. 2007]. Hegymegi et al. [2009] suggested that the capabilities of the instrument and its recording system developed by ELGI were ready for field work.

One cycle of DIDD measurement, i.e. one determination of the magnetic components, consists of 5 readings on the PPM in the following order:

- $\mathrm{F} \quad$ (total field without deflection)

- D+ (total field deflected parallel to the $\mathrm{D}$ axis)

- D- (total field deflected antiparallel to the D axis)

- I+ (total field deflected parallel to the I axis)

- I- $\quad$ (total field deflected antiparallel to the I axis)

The advances of this instrument can be summarized in the following points:

- Since the DIDD system is based on the function of a nuclear magnetometer the instrument can be qualified by the usual parameters of the nuclear magnetometers, i.e. accuracy, sampling interval etc. It also follows, that the scale factor and the offset do not need to be calibrated.

- Only the following four values have to be calibrated for the determination of the reference frame of the device [Heilig 2007]:

i) $I_{0}$ value

ii) $\mathrm{D}_{0}$ value

iii) orthogonality error of magnetic axes

iv) the leveling error of the $\mathrm{D}$ coil.

The DIDD reference frame is defined physically by the $\mathrm{D}$ coil and I coil axes. They should be orthogonal and the D coil should be horizontal, in case of the perfect alignment of the instrument. Once we know the $\mathrm{I}_{0}$ and $\mathrm{D}_{0}$ angles of a perfectly adjusted DIDD device, its reference frame is also established. Several solutions were developed for establishing the correct adjustment of the coil system [see Alldredge
1960, Csontos et. al. 2001]. The published methods were suitable to align the coil system but the continuous monitoring of the reference frame was not ensured. Further uncertainty of these methods can be the implicit assumption that the mechanical and the magnetic axes of a coil are parallel.

Mathematical solutions were also published to determine the reference frame of the DIDD system by using a DIM [Schott et al. 2001, Schott and Leroy 2001, Heilig 2007] or a pre-calibrated variometer [Heilig 2007] as an independent reference instrument. The introduction of a new method for measuring the orthogonality error of the DIDD [Heilig 2012] was another important improvement. The method for monitoring the orthogonality of the DIDD gives us the possibility to apply a way to monitor the reference frame of a DIDD by using the results of absolute measurement. We used the new practices during our repeat station survey.

\subsection{Installation of the device in the field}

The installation of the DIDD is simple provided that the gradient of the geomagnetic field is low. The device is relatively small and the suspension eliminates automatically the tilt of the pillar. Further advance is that the readings give a perfect basis for the correct orientation. The material of the coil system and other suspended parts are moulded from the mixture of epoxy resin and fibreglass. The other parts of the mechanic are made from aluminium.

Recommended steps for the complete installation:

1) Choose a hidden and shaded place near the repeat station, e.g. bush, forest or a cave etc. The distance between the DIDD and other recording sensors should be minimum 10 meters. If the spatial gradient of the geomagnetic field is high or not constant (typically in conductivity anomalous or volcanic area) try to minimize the distance between the repeat station and the DIDD site.

2) Check the gradient of the total field at the selected site to avoid the effects of any ferromagnetic materials on the measurements. Less than $3 \mathrm{nT} / \mathrm{m}$ gradient is acceptable. (Anomalous, for example basaltic, area requires special solutions, i.e. the observer has to measure the difference of the geomagnetic elements between the repeat station and the place of DIDD.)

3) Place the DIDD insturment on a nonmagnetic levelled hard plate (e.g., marble) (see Figure 1).

4) Level the instrument by using its bubbles.

5) Adjust the orientation of the coil system by observing the difference of the corresponding deflected components and test the direction of the deflected components. See the user manual [dIdD v.7.0] listed in the References for more details.

6) Cover the sensor by a water resist and temperature isolator cylinder and put a heavy nonmagnetic weight on the top of it.

7) Put the battery, the electronics and the data recording PC in a plastic box, then connect the sensor and start the 


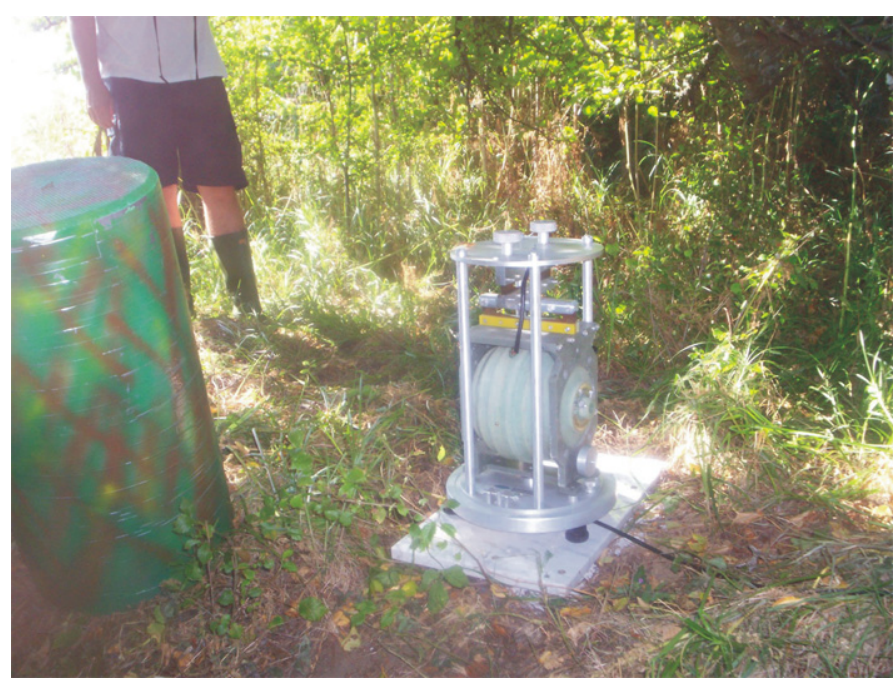

Figure 1. The DIDD device on levelled marble plate in Krbavsko Polje station. The green cylinder on the the left is the instrument case which protects the instrument against humidity and improves the temperature stability.

recording. The distance between the box and the sensor should be more than five meters.

8) After the first set of absolute measurement calculate the preliminary $\mathrm{I}_{0}$ and $\mathrm{D}_{0}$ values and modify the initialization file of the data logger.

9) Measure the orthogonality error of the DIDD. In this process the $\mathrm{D}$ and I coils have to be connected in series to ensure the same current in both coils. The current is supported by the built-in current generator of the electronics. Tune the magnetometer first to the field magnitudes to be measured. Then, record the following $5 \mathrm{~s}$ long sequences of reading: F, D+I+, D-I-, F, F, and F, D-I+, D+I-, F, F, where $\mathrm{D}+\mathrm{I}+, \mathrm{D}-\mathrm{I}-, \mathrm{D}-\mathrm{I}+, \mathrm{D}+\mathrm{I}-$ mean the total field values deflected by the I and D coils jointly in parallel or antiparallel sense. This measurement should be repeated at the end of the reoccupation.

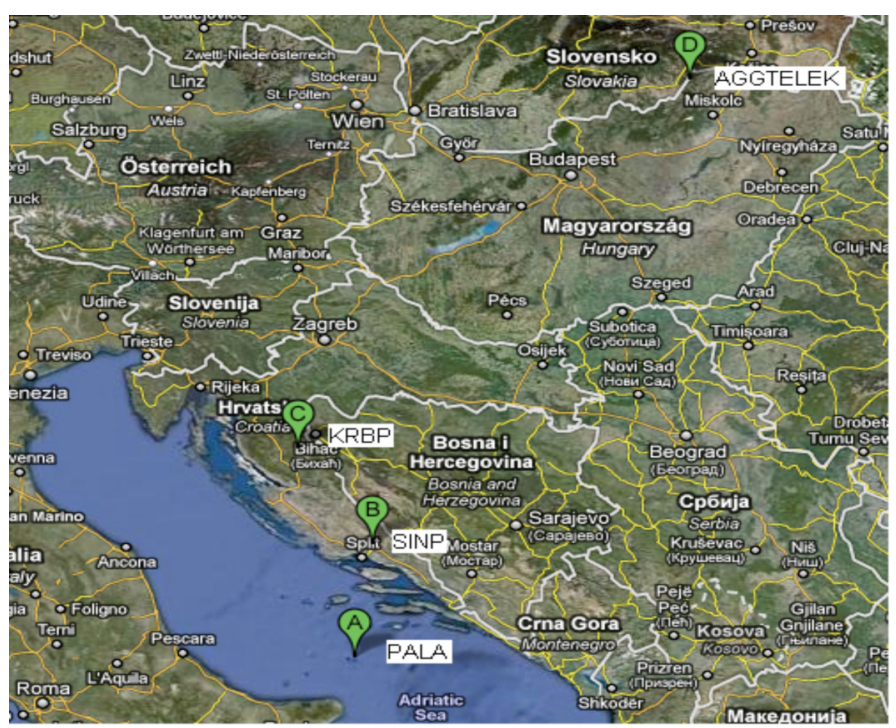

Figure 2. The repeat stations where the DIDD device was applied (from South to North PALAgruža, SINjsko Polje, KRBavsko Polje and Aggtelek).
10) In order to be able to determine the DIDD's reference from the DMI measurements (see next section) it is necessary to measure the difference of the corresponding magnetic components between the DIDD and the repeat station sites. Note, that this step can be avoided if the field gradient between the repeat station and the DIDD site is expected to be low.

The measurement can be performed in the following way: mark the original place of the DIDD and setup the DIDD instrument on a temporary site. It can be supposed that the leveling and orthogonality errors do not change significantly during the moving. Take the absolute measurements on the repeat station and on the original DIDD site, and then compute the bases of the DIDD device from each measurement. The difference between the obtained base values rely on the field difference between the original place of the DIDD and the repeat station. For that it is supposed that the reference frame of the DIDD remains stable during the absolute measurements.

\subsection{Calculation of the reference frame with comparison of} the DIM and DIDD results

If the orthogonality error of the DIDD system is known, the measured $\left(B_{i}, B_{d}, B_{s}\right)$ magnetic elements can be transformed to an orthogonal reference frame where the components are denoted by $B_{i c}, B_{d c}, B_{s c}$. Then, the ordinary $\mathrm{X}, \mathrm{Y}, \mathrm{Z}$ (North, East, vertical) magnetic components can be obtained by the following equation system [Heilig 2007]:

$$
\begin{aligned}
& X=B_{s c} \cos D_{0} \cos I_{0}-B_{d c}\left(\cos D_{0} \sin I_{0} \sin \varepsilon_{0}+\sin I_{0} \sin \varepsilon_{0}\right)- \\
& -B_{i c}\left(\cos D_{0} \sin I_{0} \cos \varepsilon_{0}-\sin D_{0} \sin \varepsilon_{0}\right) \\
& Y=B_{s c} \sin D_{0} \cos I_{0}-B_{d c}\left(\sin D_{0} \sin I_{0} \sin \varepsilon_{0}-\cos D_{0} \sin \varepsilon_{0}\right)- \\
& -B_{\text {ic }}\left(\sin D_{0} \sin I_{0} \cos \varepsilon_{0}+\cos D_{0} \sin \varepsilon_{0}\right) \\
& Z=B_{s c} \sin I_{0}+B_{d c} \cos I_{0} \sin \varepsilon_{0}+B_{i c} \cos I_{0} \cos \varepsilon_{0}
\end{aligned}
$$

where $\varepsilon_{0}, I_{0}$ and $D_{0}$ are the three unknown orientation angles of the DIDD system (see 3.1).

After determining the $\mathrm{X}, \mathrm{Y}, \mathrm{Z}$ absolute magnetic components in a certain time, $t$, and at the place of the DIDD device, the above equations are eligible to deduce the orientation angles. The absolute components are measured by DMI instrument at the repeat station pier and referenced to the DIDD site by using the previously determined component differences between the two places (see previous section).

In our work the (1) Equation system was solved by the fsolve and fminsearch functions of the GNU Octave software. In order to have a better approximation of the real orientation angles, it is recommended to use more than one set of absolute components measured at times $t_{i}$. In that case we have more equations than unknowns, and the solutions and uncertainties of the orientation angles can be obtained ac- 


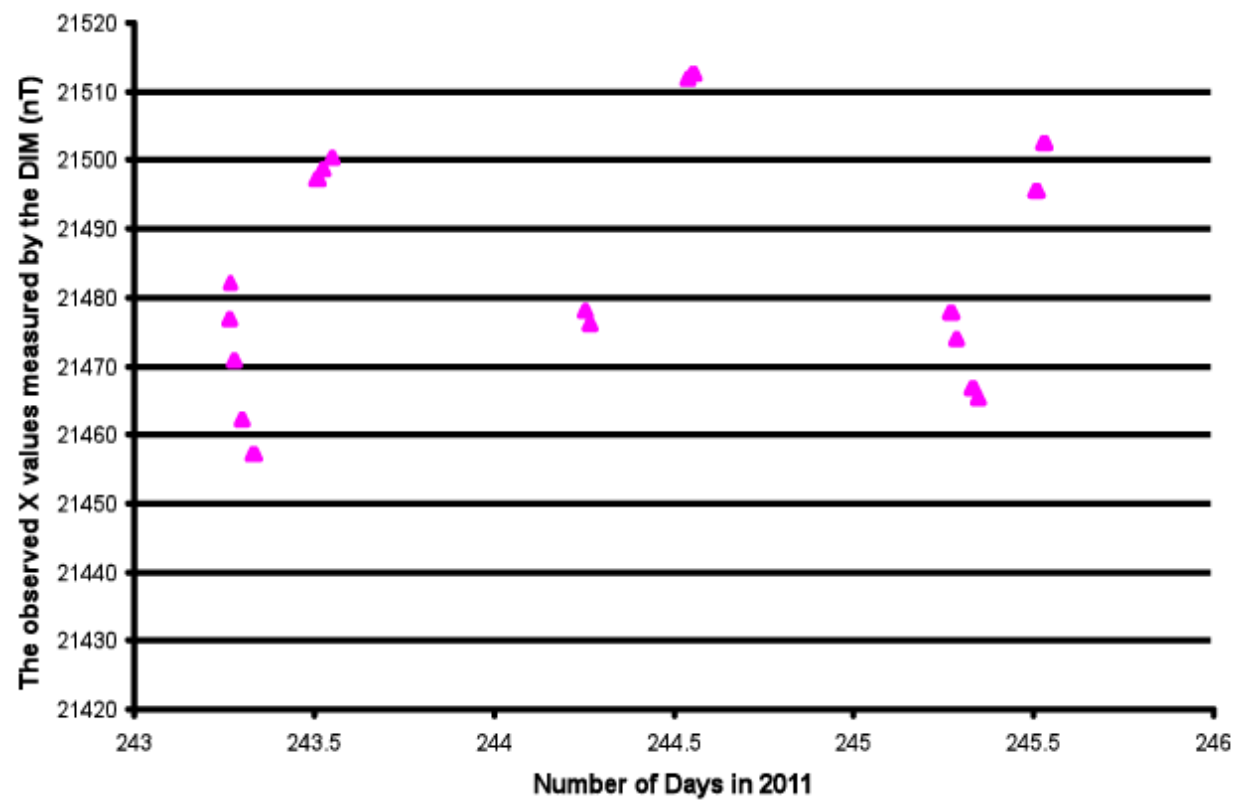

Figure 3. The absolute values of the $\mathrm{X}$ magnetic field observed on the absolute pillar of Tihany observatory in the test period of the DIDD device.

cording to the method of least squares. To increase the reliability of the numerical solutions, it is advisable to carry out the absolute measurements in those $t_{i}$ times between that the magnetic field exhibits considerable variation.

The numerical tests of the method evidenced that for the determination of the $\varepsilon_{0}$ levelling error with an accuracy of 10 arc second, the absolute components must be measured with $0.01 \mathrm{nT}$ accuracy. It is noted however, that this accuracy can be seldom achieved, especially in the case of the Y component. Moreover, the Y component is barely sensitive to the leveling error of the DIDD in comparison with the $\mathrm{X}$ and $\mathrm{Z}$ components. For this reason, it is recommended to use only the most reliable $\mathrm{X}$ and $\mathrm{Z}$ components in the (1) Equation system for the determination of the orientation parameters, if more than one series of absolute measurements are available, i.e. if more than three equations can be formulated from the results of the two components.

The certainty of a given solution can be tested by fixing the measured and computed difference of a given component below a certain limit, while allowing growing the residual of the other component without bound. Then, the constraint is applied to the other component. If the adjusted parameters do not change considerably in the two cases, the solution can be regarded reliable.

\section{Examples of real measurements - Case studies}

The methods described above enable one to calibrate the DIDD system in terrain environment. In the following, some of the real surveys carried out with the application of the portable DIDD magnetometer are briefly introduced in order to present the limit of the calibration method and suggest additional guides for the observers. Some of our experiences are related to the results of the joint Croatian-Hungarian repeat station survey carried out in 2010. Figure 2 shows the repeat stations for which the portable magnetometer was applied in the data processing.

Further experiences with the application of the portable DIDD instrument were gathered in the Tihany Geomagnetic Observatory in the year of 2011. This test provided excellent occasion for the comparison of the records of the portable DIDD and the observatory base system.

\subsection{Instrument test in the Tihany Geophysical Observatory}

The portable DIDD system was setup in the garden of the observatory. We used the instrumentation and infrastructure of the observatory (i.e., Zeiss 010A theodolite equipped by DMI D\&I one component fluxgate device and GSM-19 Overhauser magnetometer) for the absolute measurement to ensure the best quality of the test. The difference in the magnetic $\mathrm{H}$ and $\mathrm{Z}$ components measured between the absolute pillar and the DIDD site was found to be $5.5 \mathrm{nT}$ and $10 \mathrm{nT}$, respectively. (For the case of the east magnetic component we estimated $5 \mathrm{nT}$ of difference.) We used a prototype of GEM System DIDD device, that measured the orthogonality error of the coil system automatically at every midnight (see Figure 4). In order to ensure the best preconditions for the calibration of the DIDD (see previous section), the absolute measurements were performed in periods when the normal daily geomagnetic variations exhibited extreme values (see Figure 3). For the determination of the orientation values we applied the method described in Section 3.3. As a result of the long series of absolute measurements not only one set of reference frame parameters but also its temporal evolution could be monitored (Figures 5, 6, 7) for the test period. The parameters were established for noon and midnights by using two sets of absolute components ob- 

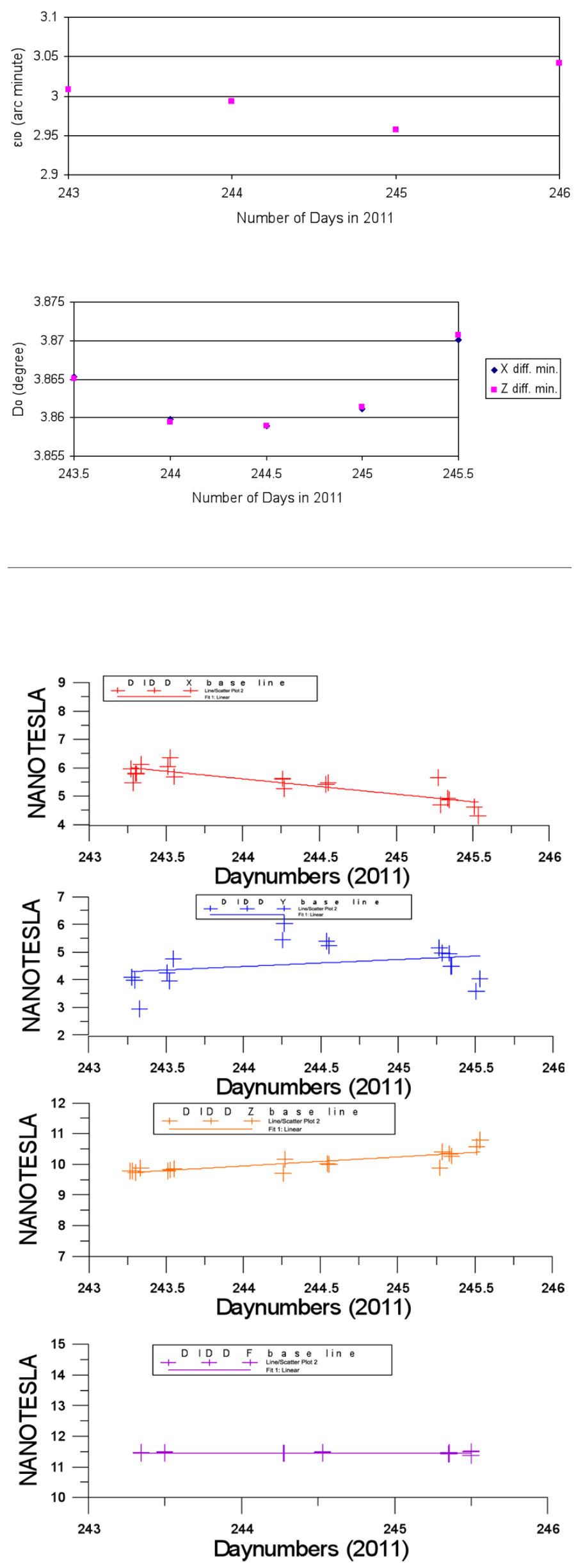

Figure 8. The adopted baseline of DIDD device in the Tihany observatory.
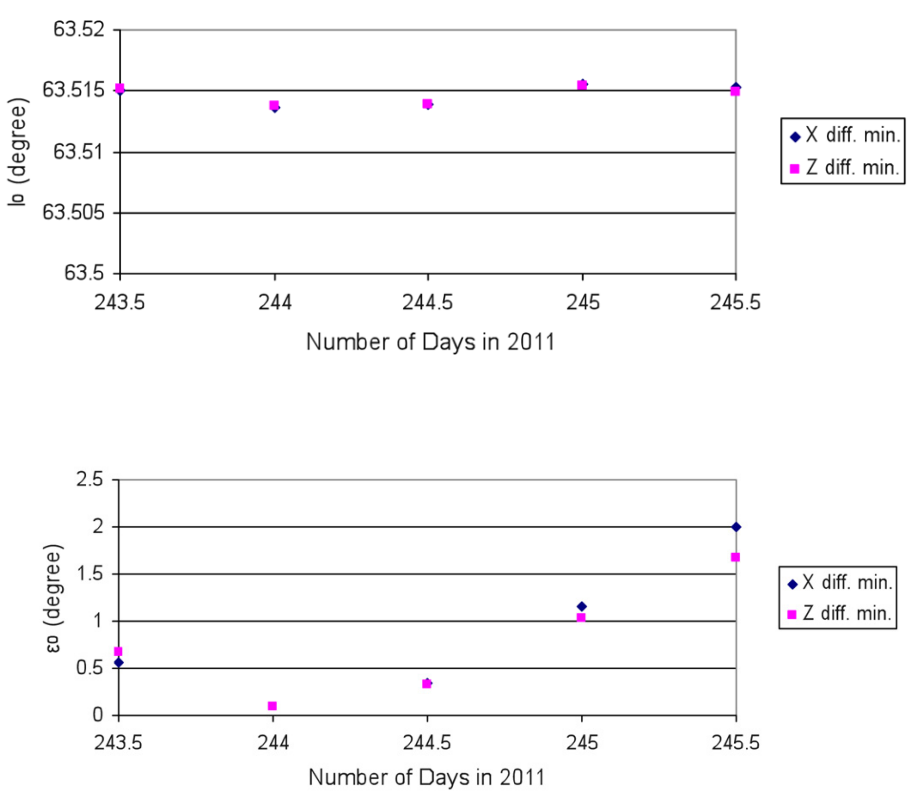

Clockwise from top left: Figure 4. The measured orthogonality error of the DIDD system; Tihany observatory 31.08.2011-02.09.2011. Figure 5. The estimated $\mathrm{I}_{0}$ parameter of the DIDD system computed with the conditions of $\left|X_{\text {meas. }}-X_{\text {comp. }}\right|<0.1 \mathrm{nT}$ (blue dots) or $\left|Z_{\text {meas. }}-Z_{\text {comp. }}\right|<0.1 \mathrm{nT}$ (pink dots); Tihany observatory 31.08.2011-02.09.2011 (see text). Figure 6. The estimated $\mathrm{D}_{0}$ parameter of the DIDD system computed with the conditions of $\left|\mathrm{X}_{\text {meas. }}-\mathrm{X}_{\text {comp. }}\right|<0.1 \mathrm{nT}$ (blue dots) or $\left|Z_{\text {meas. }}-Z_{\text {comp. }}\right|<0.1 \mathrm{nT}$ (pink dots); Tihany observatory 31.08.2011-02.09.2011 (see text). Figure 7. The estimated levelling errors of the DIDD system computed with the conditions of $\left|X_{\text {meas. }}-X_{\text {comp. }}\right|<0.1 \mathrm{nT}$ (blue dots) or $\left|Z_{\text {meas. }}-Z_{\text {comp. }}\right|<$ $<0.1 \mathrm{nT}$ (pink dots); Tihany observatory 31.08.2011-02.09.2011 (see text).

tained from consecutive morning-afternoon or afternoonmorning measuring series. The method requires high accuracy in the DMI measurements and stable magnetic field difference between the DIDD and DMI sites. The possible errors of DIM measurement or the varying spatial gradient can result in unrealistic output of the numerical iteration. Since the determination of the east magnetic component may exhibit unacceptable uncertainty, our numerical analyses were based only on the $\mathrm{X}$ and $\mathrm{Z}$ component absolute magnetic determinations. The solutions for the reference frame parameters were computed for the conditions of $\left|X_{\text {meas. }}-X_{\text {comp. }}\right|<0.1 \mathrm{nT}$ or $\left|Z_{\text {meas. }}-Z_{\text {comp. }}\right|<0.1 \mathrm{nT}$ where the meas. and comp. indices mean the measured and computed (Equation 1) component values (see previous section). The obtained results are shown on Figures 5-7 for both cases. It was concluded that the reference frame parameters were robust against the applied conditions, thus the obtained solutions were found to be reliable.

The mean values ( \pm standard deviation) of the reference frame parameters of the DIDD system calculated for all days of the Tihany test period are: $\mathrm{I}_{0}=63.5146^{\circ} \pm 0.0008^{\circ} ; \mathrm{D}_{0}=$ $3.8631^{\circ} \pm 0.0047^{\circ} ; \varepsilon_{0}=0.7764^{\circ} \pm 0.7151^{\circ} ; \varepsilon_{\mathrm{ID}}=0.05^{\circ} \pm$ $0.0157^{\circ}$.

The final baseline of the DIDD system is presented in Figure 8. 


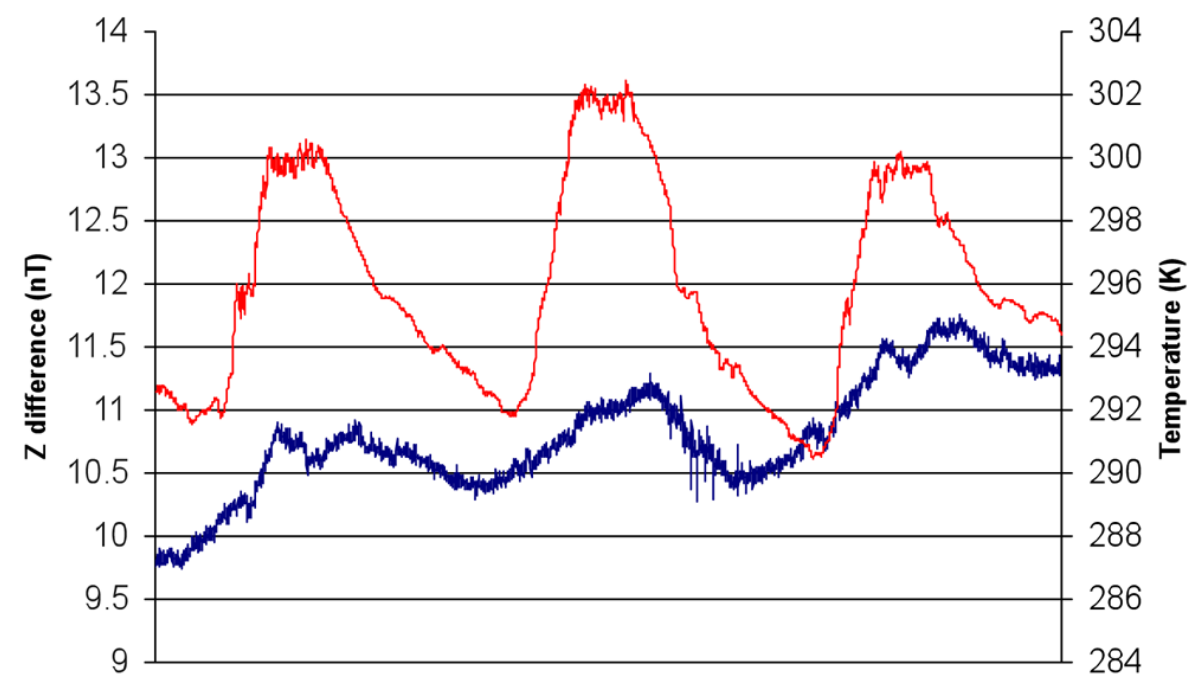

242.5242 .8243 .1243 .4243 .7244 .0244 .3244 .6244 .9245 .2245 .5

Daynumbers of 2011

Figure 9. Difference of vertical components between the preliminary data of Tihany observatory and the DIDD record (blue) and the environmental temperature (red).

It can be concluded that the baselines of the instrument fulfill the IAGA requirements [Newitt et al. 1996], i.e. the accuracy of the measurement is better than $5 \mathrm{nT}$.

The monitoring of the difference between the DIDD and observatory fluxgate records of corresponding components provides further useful information on the proper operation of the DIDD instrument. For the case of the $Z$ magnetic component the obtained difference curve is shown in Figure 9 (blue curve). The curve exhibits slow drift and fine fluctuation. The harmonic trend is more or less similar than that of the temperature variation (red curve) recorded in free air. However, the small amplitude of the harmonic variation affirms that the DIDD system has low temperature sensitivity. On the other hand the strong correlation between the red and blue (i.e., temperature and $Z$ difference) curves shows that both of the investigated magnetometers (i.e. the DIDD and the fluxgate system of the observatory) are well calibrated. (We should see more fluctuations in the difference curve which would refer to the geomagnetic variation if the instruments have calibration errors.)

4.2. The result of KRBavsko Polje repeat station in the Dinaric mountain

In the middle of July 2010 four days long reoccupation at the Krbaysko Polje (KRBP, see Figure 2) repeat station was carried out. The portable DIDD system was installed in the nearest forest, about $40 \mathrm{~m}$ away from the repeat station. The absolute instrument was a Zeiss 20A theodolite equipped by DMI D\&I one component fluxgate sensor. The total field was measured by GSM-19 magnetometer installed on an auxiliary point. A lot of series of absolute measurement
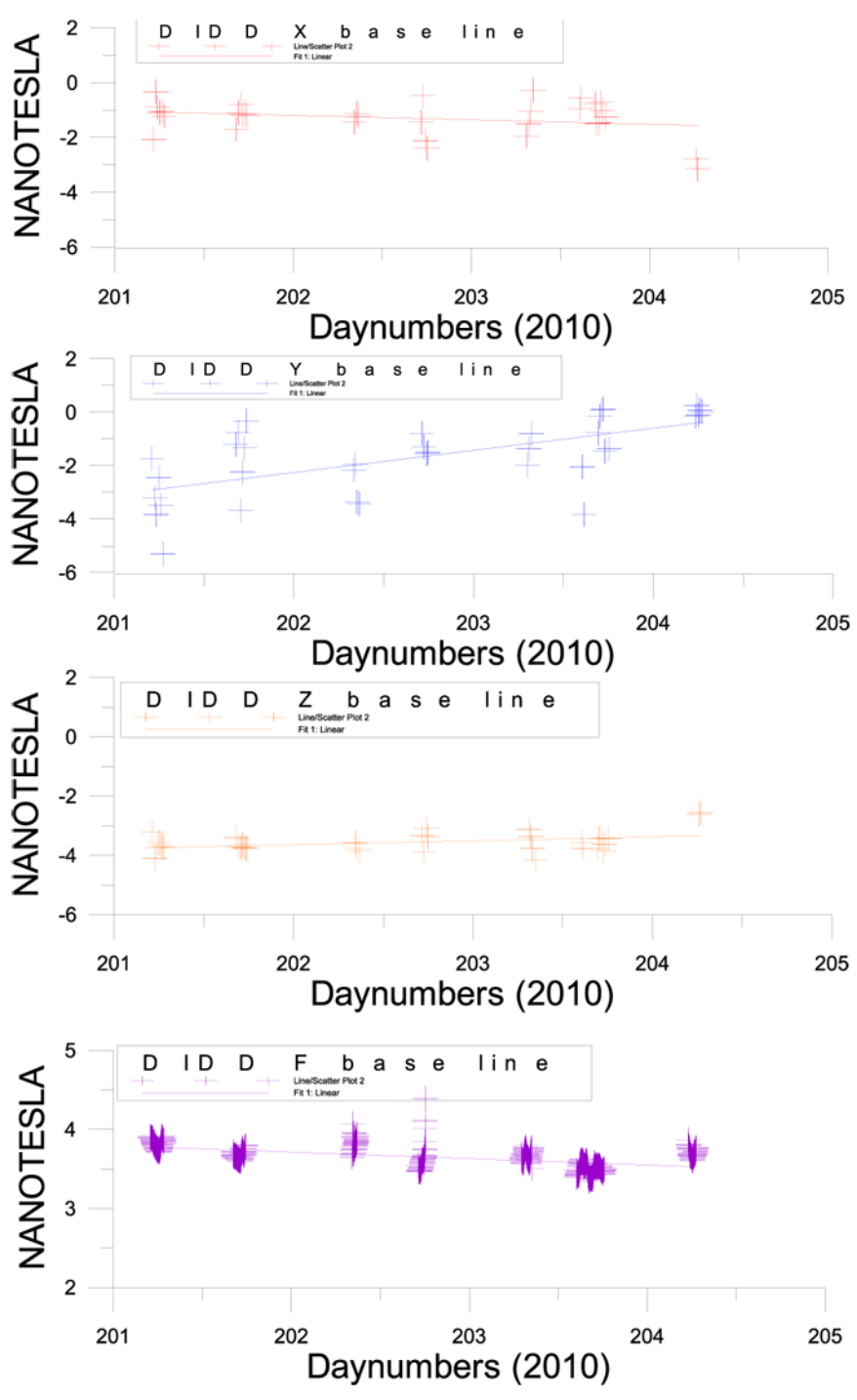

Figure 10. The adopted baseline of DIDD near the KRBP station. 


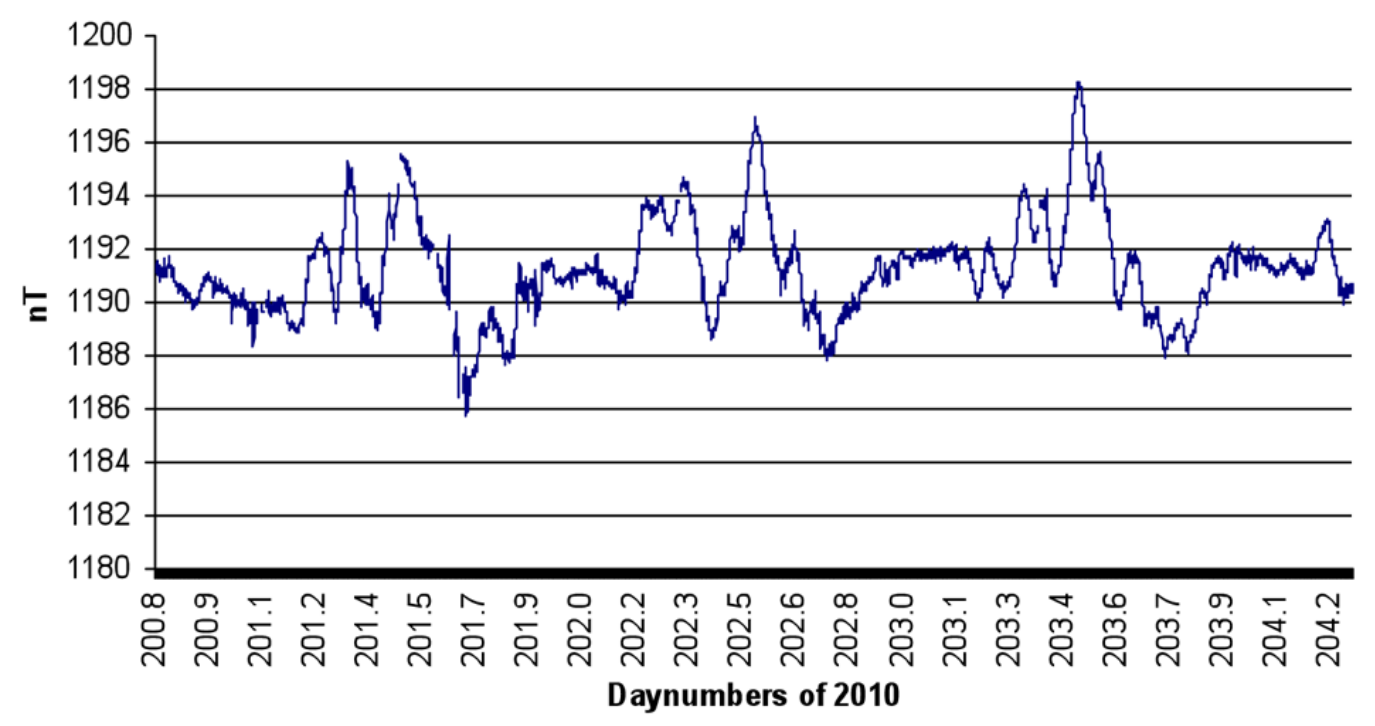

Figure 11 (above). The difference of $\mathrm{X}$ component magnetic field recorded between KRBP repeat station and Tihany observatory (minute mean values). Figure 12 (bottom page). The Y base values of the DIDD vs. the total field difference measured between the repeat station and the DIDD site at KRBP station between 20.07.2010. and 23.07.2010.

were performed in the morning and in the evening periods.

We measured the orthogonality error of the DIDD system at the beginning and at the end of the reoccupation. The average of the measured values were 3.56 and $4.02 \mathrm{arc}$ minute, thus the accepted value for $\varepsilon_{\mathrm{ID}}$ was $\varepsilon_{\mathrm{ID}}=0.063^{\circ}$ $\pm 0.0054^{\circ}$. The total field difference between the DIDD site and the repeat station was less than $4 \mathrm{nT}$. The small spatial gradient enabled us to conclude the component differences, too. (see Figure 10 for the final result). In this case we can suppose that the main difference between the sites is mostly caused by the difference of the vertical field component (induced magnetic field). This assumption is usually very close to the reality so it does not generate significant errors.

For the determination of the reference frame parameters of DIDD by using Equation 1, we selected those DIM measurements where the difference of the inclination, declination and the vertical component was maximal. Because the spatial gradient of the $\mathrm{Y}$ component was not constant, the numerical iteration was performed only with the $\mathrm{X}$ and $Z$ component measurements. The mean values of the orientation angles ( \pm standard deviation) calculated for all selected DIM results were: $\mathrm{I}_{0}=60.625^{\circ} \pm 0.0007^{\circ} ; \mathrm{D}_{0}=2.9715^{\circ}$ $\pm 0.0097^{\circ} ; \varepsilon_{0}=0.4115^{\circ} \pm 0.4333^{\circ}$. The obtained baselines of the DIDD system are shown in Figure 10.

Figure 11 shows the $\mathrm{X}$ component difference between KRBP repeat station and Tihany observatory during the three and a half day of operation of the DIDD instrument. It is seen that the peak to peak difference of the curve is about $10 \mathrm{nT}$ in the three consecutive days. On the other hand the night-time differences remain in $2 \mathrm{nT}$ wide band. It means that the quiettime component differences between the RS site and the reference observatory can be used for the final data reduction.

During the data processing it was realized that the east component baseline of the DIDD device was not stable and the difference between the total field values recorded at the site of the DIDD and the auxiliary point also showed significant fluctuations.

The characteristic of these fluctuations was more or less identical with that of the fluctuations of the horizontal geomagnetic field. A direct relation was also realized between the temporal changes of the total field differences and the east component base values of the DIDD system (see Figure 12). For the cases of the other components such relation was not evident. It suggests that the observed variation in the total field spatial gradient was mostly caused by the temporal variation of the east component spatial gradient.

One of the possible explanations for the observation of the novel temporal change of the magnetic field gradient

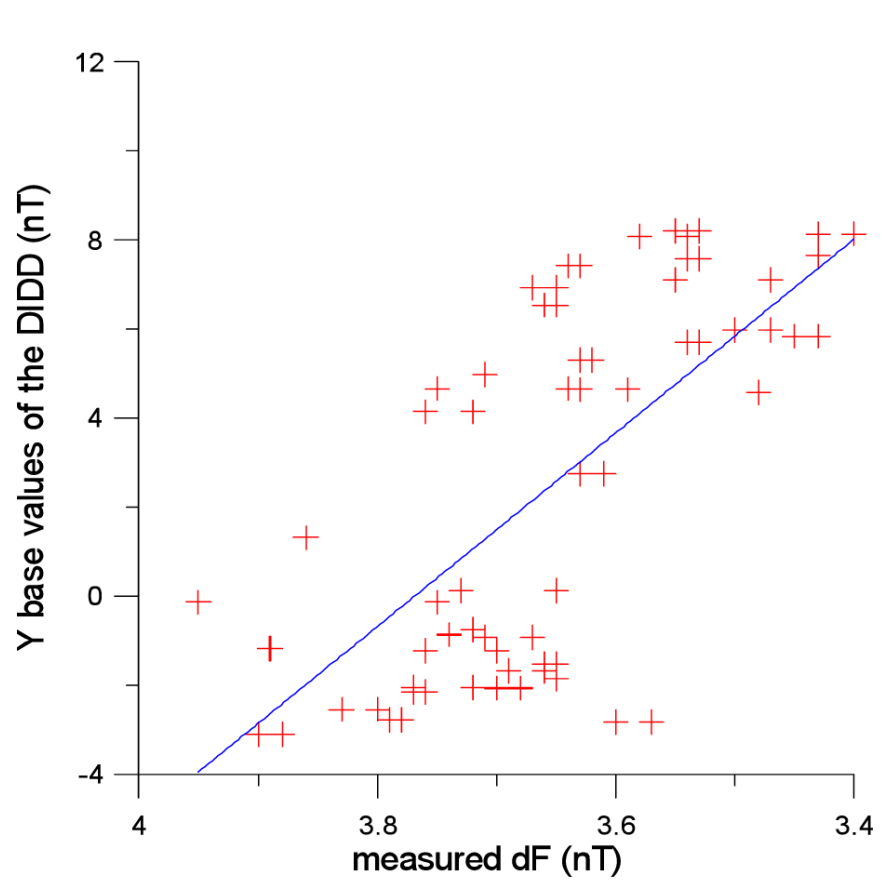


can be the significant conductivity contrast between the Adriatic sea and the mainland that can result in spatial field variation in small spatial and temporal scales, even in large distances [Soyer 2002].

This phenomenon was found to be more profound near the coast, on the Sinjsko Polje and Palagruža repeat stations (Figure 2). Here, the strong variation of the gradient field disabled the application of the method elaborated for the determination of the calibration parameters of the DIDD device and, consequently, the accurate determination of its basis. It is noted however, that even in these stations, the average of the base values determined from long series of $a b$ solute measurements provided the chance to establish accurate quiet night-time magnetic field differences between the RS-s and the reference observatories (i.e., Tihany) for the final data reduction.

\subsection{Reoccupation of the Aggtelek station (Hungary)}

On the course of the 2010 repeat station survey, the reoccupation of the Aggtelek site was carried out in November 9 and 12, 2010. The portable DIDD instrument was installed $2 \mathrm{~km}$ away from the station, in the Baradla cave, where the limestone environment and the low temperature variation provided ideal circumstances for the variometer recording. The recording started before the first and ended after the second reoccupation of the station.

Unfortunately, the scatter of the DIM results was relatively high during each of the reoccupation (see Figure 13), because the Aggtelek station was located in a muddy area, and the tripod of the theodolite could not be fixed safely during the measurements. However, we think that the means of the absolute magnetic components obtained from several series of measurements were reliable enough to deduce the
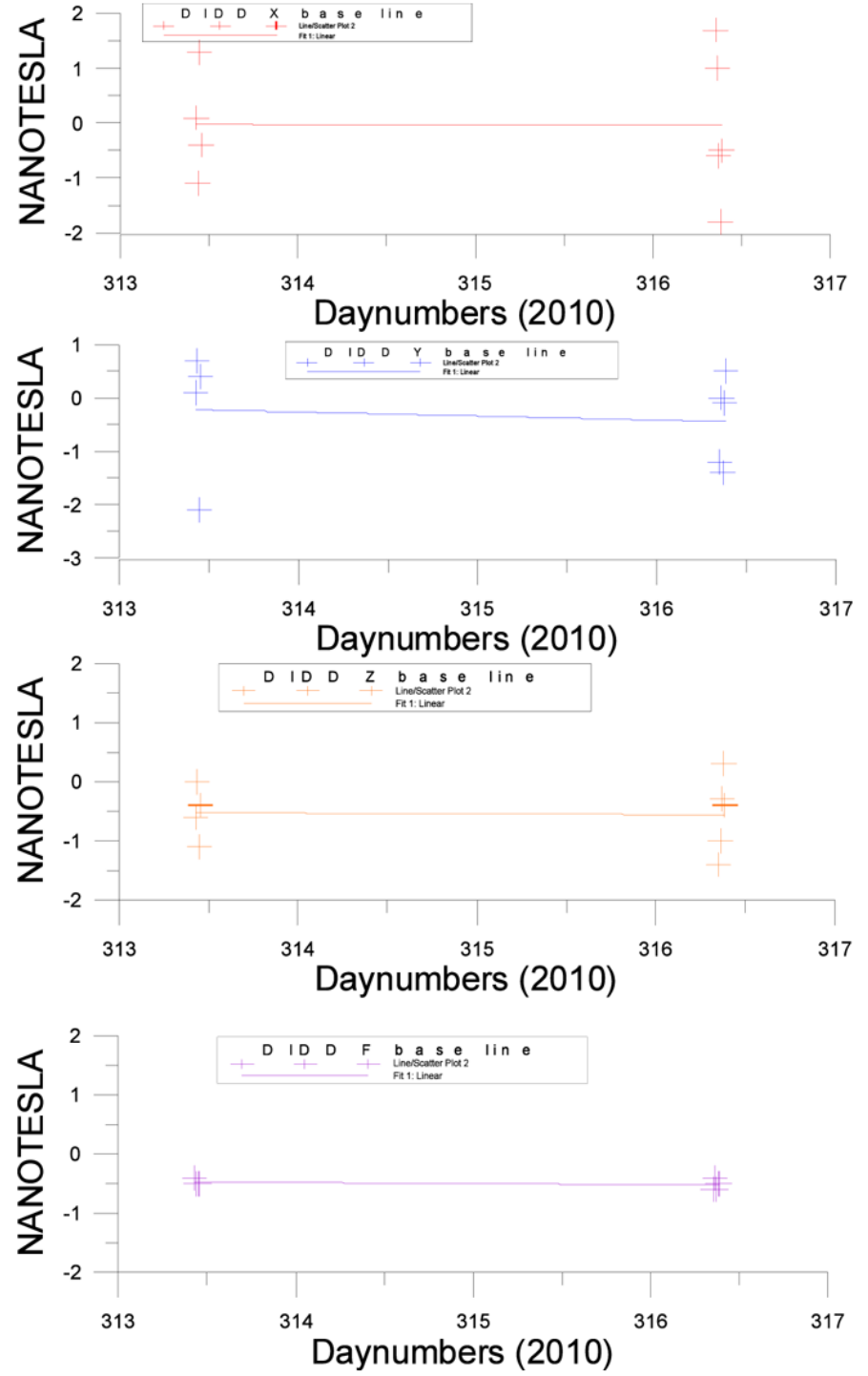

Figure 13. The adopted baseline of DIDD in the Aggtelek station. The crosses mean the bases belonging to the individual absolute measurements.
Figure 14. The difference of $X$ component magnetic field between the Aggtelek repeat station and Tihany observatory (minute mean values).

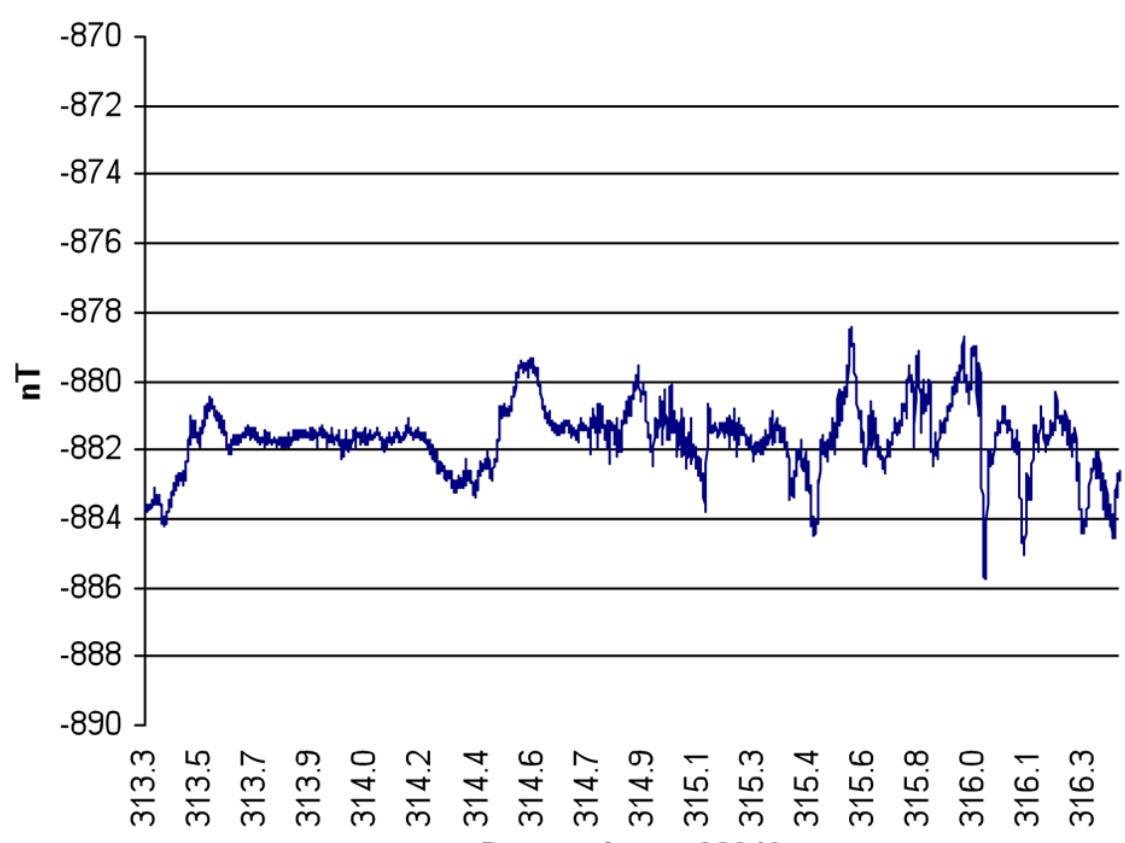

Daynumbers of 2010 
orientation values of the DIDD variometer. This conjecture was also affirmed by the adopted baselines of the DIDD (Figure 13) whose drifts exhibited less than $1 \mathrm{nT}$ of change between the two reoccupation dates, for each component. On the other hand the component differences obtained between the two reoccupations $(\mathrm{dX} \approx 18 \mathrm{nT}, \mathrm{dY} \approx-10 \mathrm{nT}, \mathrm{dZ} \approx-6 \mathrm{nT})$ were relatively low, which fact was quiet unfavorable for the numerical determination of the orientation parameters. The admitted values of the parameters were: $\varepsilon_{\mathrm{ID}}=4.51 \mathrm{arc}$ minute, $\varepsilon_{0}=18.45 \operatorname{arc}$ minute, $\mathrm{I}_{0}=65^{\circ} 9.64^{\prime}, \mathrm{D}_{0}=4^{\circ} 2.18^{\prime}$.

Finally, we present the difference of $\mathrm{X}$ component magnetic field recorded between the Aggtelek repeat station and Tihany observatory (Figure 14). Interestingly, the peak to peak variation of the difference curve was smaller in Aggtelek than in the Krbavsko Polje station against the fact that the two stations are located in a similar distance from the Tihany observatory. Moreover, during the Aggtelek survey the geomagnetic field was also disturbed by a moderate magnetic storm. It is suggested that this finding was related to the latitudinal and longitudinal locations of the two stations in comparison to reference: Aggtelek is located more towards east, Krbavsko Polje towards south form the reference observatory.

\section{Conclusions}

In this paper it was argued that the mechanical stability and low temperature sensitivity of the DIDD device provide ideal preconditions for its application as a portable device. The data recording system and the presented method of installation of the device are suitable for the perfect field application. The mathematical tools elaborated for the determination of the calibration parameters of the instrument can be efficiently applied not only in the observatory practice but also during the repeat station surveys. It was emphasized, however, that the numerical methods, when applied in RS surveys, require big stability of the spatial field gradients between the DIDD site and the repeat station pier.

Acknowledgements. This work was supported by a CroatianHungarian joint project funded by the Hungarian Science and Technology. We thank the Aggtelek National Park for the permission to install our portable variometer in the Baradla cave. The DIDD system was provided by MINGEO Ltd. and GEM Systems Inc. Geomagnetism Group of USGS is thanked for the useful remarks. We thank László Merényi for the software development of the data acquisition system of the DIDD device. Technical help in the configuration of equipment was supported by László Szabados. Balázs Heilig is specially acknowledged for valuable discussions, remarks and his participation in the reoccupation of the Aggtelek station.

\section{References}

Alldredge, L.R. (1960). A proposed automatic standard magnetic observatory, J. Geophys. Res., 65, 3777-3786.

Csontos, A., L. Hegymegi, B. Heilig and A. Körmendi (2001). The results of the delta "I" delta "D" (DIDD) measurement system at the Tihany Geomagnetic Observatory of ELGI, Contributions to Geophysics and Geodesy, 31 (1), 83-89.
Csontos, A., L. Hegymegi and B. Heilig (2007). Temperature Tests on Modern Magnetometers, Publications of the Institute of Geophysics Polish Academy of Sciences, C-99 (398), 171-177.

dIdD v7.0 (2008). Instruction Manual Release 7.4, GEM Systems, $50 \mathrm{pp}$.

Hegymegi, L., B. Heilig and A. Csontos (2004). New suspended dIdD Magnetometer for Observatory (and Field?) Use, In: Proceedings of $11^{\text {th }}$ IAGA Workshop on Geomagnetic Observatory Instruments, data acquisition and processing, Kakioka, 28-33.

Hegymegi, L., A. Csontos and B. Heilig (2009). Can we use the dIdD magnetometer on the field?, In: Proceedings of $13^{\text {th }}$ IAGA Workshop On Geomagnetic Observatory Instruments, Data Acquisition and Processing Golden and Boulder, Colorado, USA, 95-99.

Heilig, B. (2007). Intercalibration of dIdD and fluxgate magnetometers, Publications of the Institute of Geophysics Polish Academy of Sciences, C-99 (398), 144-151.

Heilig, B. (2012). Determining the orthogonality error of coil systems by means of a scalar magnetometer, Measurement Science and Technology, 23, 037001.

Jankowski, J., and C. Sucksdorff (1996). Guide for magnetic measurements and observatory practice, IAGA, Warsaw, Poland.

Korte, M. (2001). External influences in regional magnetic surveys, Contributions to Geophysics and Geodesy, 31 (1), 273-278.

Marsal, S., J.J. Curto, J.C. Riddick, J.M. Torta, O. Cid and M. Ibaňez (2009). Livingston island observatory upgrade: First results, In: Proceedings of $13^{\text {th }}$ IAGA Workshop On Geomagnetic Observatory Instruments, Data Acquisition and Processing Golden and Boulder, Colorado, USA, 154-161.

Newitt, L.R., C.E. Barton and J. Bitterly (1996). Guide for magnetic repeat station surveys, IAGA, Boulder, USA.

Schott, J.J., and P. Leroy (2001). Orientation of the DIDD magnetometer, Contributions to Geophysics and Geodesy, 31 (1), 43-50.

Schott, J.J., V. Boulard, A. Péres, J.M. Cantin and J. Bitterly (2001). Magnetic component measurement with the DIDD, Contributions to Geophysics and Geodesy, 31 (1), 35-42.

Soyer, W. (2002). Analysis of geomagnetic variations in the Central and Southern Andes, Dissertation zur Erlangung des Doktorgrades im Fachbereich Geowissenschaften an der Freien Universität Berlin.

\footnotetext{
${ }^{\star}$ Corresponding author: András Csontos,

Eötvös Loránd Geophysical Institute, Budapest, Hungary; email: csontos@elgi.hu.
}

(C) 2012 by the Istituto Nazionale di Geofisica e Vulcanologia. All rights reserved. 